\title{
Shannon Entropy in Confined He-Like Ions within a Density Functional Formalism
}

\author{
Sangita Majumdar and Amlan K. Roy * \\ Department of Chemical Sciences, Indian Institute of Science Education and Research (IISER) Kolkata, \\ Mohanpur-741246, Nadia, WB, India; sm15rs054@iiserkol.ac.in \\ * Correspondence: akroy@iiserkol.ac.in or akroy6k@gmail.com
}

Received: 6 January 2020; Accepted: 4 February 2020; Published: 10 February 2020

\begin{abstract}
Shannon entropy in position $\left(S_{\mathbf{r}}\right)$ and momentum $\left(S_{\mathbf{p}}\right)$ spaces, along with their sum $\left(S_{t}\right)$ are presented for unit-normalized densities of $\mathrm{He}_{\mathrm{Li}} \mathrm{Li}^{+}$and $\mathrm{Be}^{2+}$ ions, spatially confined at the center of an impenetrable spherical enclosure defined by a radius $r_{c}$. Both ground, as well as some selected low-lying singly excited states, viz., 1 sns $(n=2-4){ }^{3} \mathrm{~S}, 1 \mathrm{snp}(\mathrm{n}=2-3){ }^{3} \mathrm{P}, 1 \mathrm{~s} 3 \mathrm{~d}{ }^{3} \mathrm{D}$, are considered within a density functional methodology that makes use of a work function-based exchange potential along with two correlation potentials (local Wigner-type parametrized functional, as well as the more involved non-linear gradient- and Laplacian-dependent Lee-Yang-Parr functional). The radial Kohn-Sham (KS) equation is solved using an optimal spatial discretization scheme via the generalized pseudospectral (GPS) method. A detailed systematic analysis of the confined system (relative to the corresponding free system) is performed for these quantities with respect to $r_{c}$ in tabular and graphical forms, with and without electron correlation. Due to compression, the pattern of entropy in the aforementioned states becomes characterized by various crossovers at intermediate and lower $r_{c}$ regions. The impact of electron correlation is more pronounced in the weaker confinement limit and appears to decay with the rise in confinement strength. The exchange-only results are quite good to provide a decent qualitative discussion. The lower bounds provided by the entropic uncertainty relation hold well in all cases. Several other new interesting features are observed.
\end{abstract}

Keywords: Shannon entropy; quantum confinement; impenetrable boundary; excited states; helium-like ions; exchange correlation

\section{Introduction}

A particle in an impenetrable box of infinite height has served the role of a simple, elegant pedagogical tool to illustrate the effects of boundary condition on the energy spectrum of a quantum system. Understanding of such a system in some sub-region $\Omega$ of space (in contrast to the "whole" space available in a free system) offers new insights to simulate realistic situations in highly inhomogeneous media or in an external field. Matter constricted under such an extreme pressure environment gives rise to a wide range of novel changes (from the respective free counterpart) in energy spectra, electronic structure, chemical reactivity, ionization potential, polarizability etc., depending on the geometrical forms of the cavity and dimensions. This has inspired a variety of theoretical and experimental works. Some prominent applications are found in the context of the cell model of a liquid, superlattice structure, quantum dot, quantum wire, atoms/molecules encapsulated inside nanocavities (like fullerene, zeolite sieves, porous silicon, carbon nanotube), modeling defects in solids, confined phonons (or plasmons, polaritons, gas of bosons), as well as astrophysical phenomena such as the mass-radius relation of white dwarfs, ionized plasma etc. The topic is vast, and there has been a burgeoning growth of activity as evident from an extensive literature having many excellent 
comprehensive reviews. The interested reader may refer to the following reviews [1-5] and the references therein.

The first report of a confined hydrogen atom (CHA) within a sphere having rigid impenetrable walls was published as early as in 1937 [6], imposing the Dirichlet boundary condition that the wave function vanishes at the boundary. Subsequently, many attempts have been made to estimate the eigenvalues and eigenfunctions invoking a wide range of approximate analytic, semi-analytic, and purely numerical schemes. Here we mention a few like Rayleigh-Schrödinger perturbation theory, the Wentzel-Kramers-Brillouin method, the power-series solution, hypervirial theorem, Padé approximation, Lie algebraic treatment, super-symmetric quantum mechanics, the Lagrange-mesh method, the asymptotic iteration method, searching the zeros of the hypergeometric function, the generalized pseudospectral (GPS) method and the Hartree-Fock (HF) method [7-22]. In recent years, the exact solution of the Schrödinger equation has been found in terms of the Kummer-M function (confluent hypergeometric) [16]. As the boundary approaches the nucleus and the volume of confinement squeezes, one notices a monotonic increase in energy in CHA. Another interesting feature is that, in contrast to a free atom, due to the breaking of the symmetry in CHA, it is characterized by different energy eigenvalues, eigenfunctions, and reduced degeneracies. On the other hand, new degeneracies, namely simultaneous, incidental and inter-dimensional degeneracy, which are non-existent in the free system, are introduced in CHA afresh. Apart from the effect of compression on the ground and various energy levels, properties such as the dipole shielding factor, nuclear magnetic screening constant, hyper-fine splitting constant, pressure, static and dynamic polarizability, etc., were examined.

An analogous study of the compressed He atom is a prototypical non-trivial mathematical problem. Due to the presence of inter-electronic repulsion, the SU(3) symmetry of the simplified one-electron case is broken, which promises many exciting physics. Ever since the variational calculation of energy variation [23] with respect to the cage radius and function of pressure, vigorous attempts have been known. Some of them include the Roothaan-HF-type calculation with the Slater-type basis [24] or with its modifications [25], configuration interaction [26,27], quantum Monte Carlo [28], a host of direct variational schemes with the appropriate choice of cut-off function [8,29-31], variational method with B-splines basis [32], and Rayleigh-Schrödinger perturbation theory $[30,33]$. Some other prominent works are explicitly correlated Hylleraas-type wave functions within the variational framework [34-41], a combination of the quantum genetic algorithm and HF method [42], variational Monte Carlo [43,44], HF calculation employing local and global basis sets [45], and so on. Whereas a vast majority of publications have focused on the lowest state, low-lying excited states were also treated quite decently. For example, 1 sns ${ }^{1,3} \mathrm{~S}$ states in $[29,30,35,38-42,44,46], 1 \mathrm{~s} 2 \mathrm{p}^{3} \mathrm{P}$, ${ }^{1} \mathrm{P}$ states in $[29,42,46]$, singly excited $1 \mathrm{~s} 3 \mathrm{~d}{ }^{3} \mathrm{D},{ }^{1} \mathrm{D}$ and some doubly excited states in $[42,46]$ etc., using a host of theoretical approaches giving results of varied accuracy.

All the above works pertain to the wave function-based methods. In the past two decades, some results have been published within the alternative density-based concept, the so-called density functional theory (DFT) [47-49]. Thus, within an exchange-only framework (using two exchange functionals, viz. local density approximation (LDA) [47] and Becke-88 exchange potential [50]), the desired Kohn-Sham (KS) equation was solved satisfying the Dirichlet boundary condition for many-electron systems via the numerical shooting method [51]. The usefulness of a one-parameter hybrid exchange functional (including a fraction of exact exchange and the Perdew-Burke-Ernzerhof functional) for treatment of confined atoms has been presented recently [52]. In another attempt, ground and $1 \mathrm{~s} 2 \mathrm{~s}{ }^{3} \mathrm{~S},{ }^{1} \mathrm{~S}$ states of a confined He atom were reported [53] taking into account the LDA-approximated exchange-correlation (XC) (with Perdew-Wang parametrization for correlation [54]) with and without self-interaction correction. Response properties such as polarizability and hyperpolarizability of the confined He atom were reported within a DFT-based variation-perturbation approach [55]. In a recent work [56], spherically confined atoms were treated by means of the local exchange potential corresponding to the Zhao-Morrison-Parr and Becke-Johnson potential. Moreover, 
spherical confinement was used in the comparative study (taking the free-ion limit as reference) of the behavior of spin potential and the pairing energy of first row transition metal cations within the KS model [57]. A detailed analysis of the correlation energy, the performance of several commonly used functionals, electron density, as well as the XC potential in some constrained atoms have been reported [58,59]. The calculation of the static polarizability of confined $\mathrm{He}$ and $\mathrm{Ne}$ atoms was done through time-dependent DFT in [60].

Recently, there has been a growing interest in information theoretical analysis of diverse models and realistic systems. They have found wide-spread applications in many branches of physics and chemistry, such as thermodynamics, spectroscopy, quantum mechanics. In chemical physics, typically they can provide valuable information regarding the localization-delocalization, diffusion of atomic orbital, periodic properties, spreading of electron density, correlation energy, etc. Entropic uncertainty measures based on these quantities are arguably the most effective quantifiers of uncertainty, as they do not relate to any specific points of the respective Hilbert space. The present work is particularly concerned with Shannon entropy $(S)$ [61,62], which is the arithmetic mean of uncertainty. Interestingly, $S$ like some of the other measures such as Fisher information, Onicescu energy and Rényi entropy are functionals of density and also characterize density. Many articles have been published to analyze these measures in free systems (e.g., for free He, we refer the reader to a recent article [63]), but in confined quantum systems as treated here, analogous studies are quite limited. Two such reports $[64,65]$ in CHA are available so far. A systematic variation of $S$ with respect to $r_{c}$ in $r$ and $p$ spaces has been presented only recently $[66,67]$ for $\ell=0$, as well as non-zero- $\ell$ states. One finds that confinement affects $S$ more profoundly in the stronger regime. Further, $S_{\mathbf{r}}$ increases with the rise in $r_{c}$ and at the very low- $r_{c}$ region $(\approx 0.1)$, CHA displays exactly the opposite trend from a free $\mathrm{H}$ atom $\left(S_{\mathrm{r}}\right.$ declines with the rise in $n$ keeping $l$ fixed). Usually, the effect of perturbation on higher quantum number states is more pronounced. In confined two-electron isoelectronic series $\left(\mathrm{H}^{-}, \mathrm{He} \mathrm{Li}^{+}, \mathrm{Be}^{2+}\right), \mathrm{S}$ has been reported for only ground states [64] by means of BLYP calculations.

Some reports [52,68-73] are available on $S$ in penetrable confinement in atoms. For example, it was observed [68] that, in CHA, up to a certain value of $r_{c}, S_{\mathrm{r}}$ decreases with $r_{c}$. However, for small $r_{c}$ and depending on barrier height, $S_{\mathbf{r}}$ may also increase. Apart from constant potential, it was probed [73] for confinements imposed by a dielectric continuum and by isotropic harmonic potential. It was also proposed [72] as an indicator to measure the delocalization of electron density. Ground-state atomic $S^{\prime}$ s, as a function of the width of confining potential, was calculated by employing the correlated Hylleraas-type wave function in both repulsive and attractive finite potentials [70]. Confinement by an inert geometric planar boundary with finite barrier height has been studied [69] within a Thomas-Fermi-Dirac-Weizsäcker-type DFT framework. Some limited works exist on excited states as well, viz. low-lying singly [70] and doubly [71] excited states. It is worth noting that $S$ values in He are available [74] in selected excited states, such as ${ }^{1,3} S^{e}, 1,3 P^{o}$ and ${ }^{1,3} D^{e}$.

The objective of this work is to make a thorough systematic analysis of $S$ in a He-like ion placed inside a spherical cage or radius $r_{c}$. This is done by invoking DFT within a work function-based exchange potential in conjunction with two correlation functionals, viz., a local, parametrized Wigner-type [75] and somewhat involved nonlinear Lee-Yang-Parr (LYP) [76] functional. The pertinent KS differential equation is solved within the Dirichlet boundary condition by means of the GPS method in an accurate and efficient manner. The electron density as well as $S_{r}$ are calculated from the self-consistent orbitals. The $p$-space orbitals are constructed from respective $r$-space orbitals via standard Fourier transform, from which the $S_{p}$ 's are computed. Variation of $S_{\mathbf{r}}, S_{\mathbf{p}}$ and the total Shannon entropy sum $\left(S=S_{\mathbf{r}}+S_{\mathrm{p}}\right)$ with respect to $r_{c}$ is offered for $\mathrm{He}, \mathrm{Li}^{+}$and $\mathrm{Be}^{2+}$. Apart from the ground state, we also consider singly excited ${ }^{3} \mathrm{~S},{ }^{3} \mathrm{P}$ and ${ }^{3} \mathrm{D}$ states arising out of configurations $1 \mathrm{sns}$ $(\mathrm{n}=2-4)^{3} \mathrm{~S}, 1 \mathrm{snp}(\mathrm{n}=2-3)^{3} \mathrm{P}$ and 1 snd $(\mathrm{n}=3)$. As is apparent from the preceding discussion, there is a lack of such results in the literature, especially in excited states, and we attempt to provide them. The article is organized as follows. Section 2 outlines the methodology used. Section 3 discusses the 
results along with a comparison with available references, while Section 4 makes a few concluding remarks.

\section{Methodology}

Here, we briefly outline the proposed density functional method for ground and excited states of an arbitrary atom centered inside an impenetrable spherical cavity, followed by the GPS method for the calculation of the eigenvalues and eigenenergies of the corresponding KS equation. It may be noted that the present method has been very successfully used for ground and various excited states (such as singly, doubly, triply excited states corresponding to low- and high-lying excitation, valence and core excitation, autoionizing states, hollow and doubly hollow states, very high-lying Rydberg states, satellites states, etc.) of free or unconfined neutral atoms, as well as positive and negative ions in a series of articles [77-83]. However, it has never been tested for any confinement studies as intended here. Thus, we present an extension of the method for the purpose of confinement effects. Our focus remains on essential portions, omitting the relevant details, which could be found in the above references.

The starting point is the non-relativistic single-particle time-independent KS equation with imposed confinement, which can be conveniently written as (atomic unit employed unless otherwise mentioned),

$$
\mathbf{H}(\mathbf{r}) \phi_{i}(\mathbf{r})=\epsilon_{i}(\mathbf{r}) \phi_{i}(\mathbf{r}),
$$

where $\mathbf{H}$ is the perturbed KS Hamiltonian, written as,

$$
\begin{aligned}
\mathbf{H}(\mathbf{r}) & =-\frac{1}{2} \nabla^{2}+v_{e f f}(\mathbf{r}) \\
v_{e f f}(\mathbf{r}) & =v_{n e}(\mathbf{r})+\int \frac{\rho\left(\mathbf{r}^{\prime}\right)}{\left|\mathbf{r}-\mathbf{r}^{\prime}\right|} \mathrm{d} \mathbf{r}^{\prime}+\frac{\delta E_{x c}[\rho(\mathbf{r})]}{\delta \rho(\mathbf{r})}+v_{c o n f}(\mathbf{r}) .
\end{aligned}
$$

In the above, $v_{n e}(\mathbf{r})$ and $v_{\text {conf }}(\mathbf{r})$ signify external electron-nuclear attraction and the effective confining potentials, whereas the second and third terms in the right-hand side denote classical Coulomb (Hartree) repulsion and many-body XC potentials, respectively. The desired confinement effect is built into the system by introducing a potential of the following form $\left(r_{c}\right.$ refers to the radius of a spherical enclosure),

$$
v_{\text {conf }}(\mathbf{r})= \begin{cases}0, & r \leq r_{c} \\ +\infty, & r>r_{c}\end{cases}
$$

Though DFT has achieved impressive success in explaining the electronic structure and properties of a many-electron system in the ground state in the past four decades, the calculation of excited-state energies and densities has remained a bottleneck. This is mainly due to the absence of a Hohenberg-Kohn theorem parallel to the ground state, as well as the lack of a suitable XC functional valid for a general excited state. In this work, we employed an accurate work function-based exchange potential, which was physically motivated $[84,85]$. Accordingly, exchange energy is interpreted as the interaction energy between an electron at $\mathbf{r}$ and its Fermi-Coulomb hole charge density $\rho_{x}\left(\mathbf{r}, \mathbf{r}^{\prime}\right)$ at $\mathbf{r}^{\prime}$, and given by,

$$
E_{x}[\rho(\mathbf{r})]=\frac{1}{2} \iint \frac{\rho(\mathbf{r}) \rho_{x}\left(\mathbf{r}, \mathbf{r}^{\prime}\right)}{\left|\mathbf{r}-\mathbf{r}^{\prime}\right|} \mathrm{d} \mathbf{r} \mathrm{d} \mathbf{r}^{\prime}
$$


Assuming that a unique local exchange potential $v_{x}(\mathbf{r})$ exists for a given state, it can be defined as the work done in bringing an electron to the point $\mathbf{r}$ against the electric field generated by its Fermi-Coulomb hole density, leading to the following form,

$$
v_{x}(\mathbf{r})=-\int_{\infty}^{r} \mathcal{E}_{x}(\mathbf{r}) \mathrm{d} l
$$

where the electric field is expressed as,

$$
\mathcal{E}_{x}(\mathbf{r})=\int \frac{\rho_{x}\left(\mathbf{r}, \mathbf{r}^{\prime}\right)\left(\mathbf{r}-\mathbf{r}^{\prime}\right)}{\left|\mathbf{r}-\mathbf{r}^{\prime}\right|^{3}} \mathrm{~d} \mathbf{r}^{\prime}
$$

The Fermi hole can be written in terms of orbitals as,

$$
\rho_{x}\left(\mathbf{r}, \mathbf{r}^{\prime}\right)=-\frac{\left|\gamma\left(\mathbf{r}, \mathbf{r}^{\prime}\right)\right|^{2}}{2 \rho(\mathbf{r})}
$$

where $\left|\gamma\left(\mathbf{r}, \mathbf{r}^{\prime}\right)\right|=\sum_{i} \phi_{i}^{*}(\mathbf{r}) \phi_{i}\left(\mathbf{r}^{\prime}\right)$ is the single particle density matrix and $\rho(\mathbf{r})$ is the electron density, expressed in terms of occupied atomic orbitals ( $n_{i}$ denotes occupation number) as,

$$
\rho(\mathbf{r})=\sum_{i=1}^{N} n_{i}\left|\phi_{i}(\mathbf{r})\right|^{2}
$$

While the exchange potential $v_{x}(\mathbf{r})$ corresponding to a given state arising from an electronic configuration can be accurately calculated by the above procedure as delineated, the correlation potential $v_{\mathcal{C}}(\mathbf{r})$ is unknown and must be approximated for practical calculations. The current work incorporates two correlation functionals, namely a Wigner-type [75] and LYP [76]. These two functionals were chosen on the basis of their success in the unconfined atomic excited states, which were recorded in [77-83]. This work will help shed some light on the applicability of such functionals in the context of confined quantum systems, including those studied here.

With this choice of $v_{x}(\mathbf{r})$ and $v_{\mathcal{C}}(\mathbf{r})$, the resulting KS differential equation,

$$
\left[-\frac{1}{2} \nabla^{2}+v_{e f f}(\mathbf{r})\right] \phi_{i}(\mathbf{r})=\varepsilon_{i} \phi_{i}(\mathbf{r}),
$$

needs to be solved, where $v_{e f f}(\mathbf{r})$ is as defined in Equation (2), maintaining the Dirichlet boundary condition. For an accurate and efficient solution, we adopted the GPS scheme leading to a non-uniform, optimal spatial discretization. It is a simple, but very effective method; its success has been demonstrated for many static and dynamic properties of a variety of singular and non-singular potentials of physical and chemical interest [80-83,86-89] such as, Coulomb, Húlthen, Yukawa, logarithmic, spiked oscillator, Hellmann potential, etc., along with its recent extension to quantum confinement $[15,21,22]$. As the method is very well established and documented, in the following, we will mention a very brief summary of it; the details are available in the cited references. 
The key characteristic of this approach is to approximate an exact function $f(x)$ defined in the interval $[-1,1]$ by an $N^{\text {th }}$-order polynomial $f_{N}(x)$,

$$
f(x) \cong f_{N}(x)=\sum_{j=0}^{N} f\left(x_{j}\right) g_{j}(x),
$$

which ensures that the approximation is exact at the collocation points $x_{j}$,

$$
f_{N}\left(x_{j}\right)=f\left(x_{j}\right) .
$$

Here, we utilize the Legendre pseudo-spectral method where $x_{0}=-1, x_{N}=1$, while $x_{j}(j=$ $1, \ldots, N-1)$ 's are defined by the roots of the first derivative of Legendre polynomial $P_{N}(x)$, with respect to $x$, namely,

$$
P_{N}^{\prime}\left(x_{j}\right)=0 .
$$

In Equation (10), $g_{j}(x)$ are termed cardinal functions, and as such are expressed as,

$$
g_{j}(x)=-\frac{1}{N(N+1) P_{N}\left(x_{j}\right)} \frac{\left(1-x^{2}\right) P_{N}^{\prime}(x)}{\left(x-x_{j}\right)},
$$

fulfilling the unique property that $g_{j}\left(x_{j^{\prime}}\right)=\delta_{j^{\prime}, j}$. Then, use of a non-linear mapping followed by a symmetrization procedure eventually leads to a symmetric eigenvalue problem, which is solved by standard available software to provide accurate eigenvalues and eigenfunctions.

The $p$-space wave function is obtained numerically from the Fourier transform of the respective $r$-space counterpart in the following way,

$$
\xi(\mathbf{p})=\left(\frac{1}{2 \pi}\right)^{3 / 2} \int \phi(\mathbf{r}) e^{i \mathbf{p} \cdot \mathbf{r}} \mathrm{dr} .
$$

It is noted here that $\xi(\mathbf{p})$ is not normalized; hence, it needs to be normalized. The normalized $r$ - and $p$-space densities are represented as $\rho(\mathbf{r})=\sum_{i=1}^{N} n_{i}\left|\phi_{i}(\mathbf{r})\right|^{2}$ and $\Pi(\mathbf{p})=\sum_{i=1}^{N} n_{i}\left|\xi_{i}(\mathbf{p})\right|^{2}$, respectively, where $n_{i}$ represents the occupation number of each orbital.

Next $S_{\mathbf{r}}, S_{\mathbf{p}}$, and Shannon entropy sum $S_{t}$ are defined in terms of the expectation values of logarithmic probability density functions, which have the forms given below as,

$$
\begin{aligned}
& S_{\mathbf{r}}=-\int_{\mathcal{R}^{3}} \rho(\mathbf{r}) \ln [\rho(\mathbf{r})] \mathrm{d} \mathbf{r}, \quad S_{\mathbf{p}}=-\int_{\mathcal{R}^{3}} \Pi(\mathbf{p}) \ln [\Pi(\mathbf{p})] \mathrm{d} \mathbf{p}, \\
& S_{t}=\left[S_{\mathbf{r}}+S_{\mathbf{p}}\right] \geq 3(1+\ln \pi), \quad \text { in } 3 \text { dimension. }
\end{aligned}
$$

Here, $\rho(\mathbf{r})$ and $\Pi(\mathbf{p})$ are both normalized to unity.

All the computations are done numerically. The convergence is ensured by carrying out calculations with respect to variation in grid parameters, such as the total number of radial points and the maximum range of the grid. It is generally observed that convergence is achieved relatively easily in the lower $r_{c}$ region compared to the $r_{c} \rightarrow \infty$ limit. All results given in the following tables and plots were checked for the above convergence. 


\section{Result and Discussion}

At the onset, it would be appropriate to mention a few points to facilitate the discussion. The net information measures in $r$ and $p$ space of confined many-electron system consist of (i) radial and (ii) angular contributions. The angular part remains invariant in both spaces with respect to change in boundary condition resulting from confinement. Note that, an analogous energy analysis with respect to $r_{c}$ in these and other excited states, would be presented elsewhere. However, it suffices here to mention that, energies for both ground and excited states obtained with the present method are in good agreement with those available in literature. Here our aim is to investigate $S$ for confined two-electron atomic systems at various $r_{c}$ in several low-lying states. These are calculated for spherically confined $\mathrm{He}$ and extended to confined iso-electronic members, namely, $\mathrm{Li}^{+}$and $\mathrm{Be}^{2+}$. Apart from ground state, following low-lying singly excited states have been considered, viz., $1 \mathrm{sns}^{3} \mathrm{~S}$ with $\mathrm{n}=2-4 ; 1 \mathrm{snp}{ }^{3} \mathrm{P}$ with $\mathrm{n}=2-3 ; 1$ snd ${ }^{3} \mathrm{D}$ having $\mathrm{n}=3$. The necessary results are presented in the following tables and plots along with available literature values, for a comparative discussion. All calculations are performed with unit-normalized density.

To start with, Table 1 imprints the numerical results of $S_{\mathbf{r}}, S_{\mathbf{p}}, S_{t}$ for confined $\mathrm{He}, \mathrm{Li}^{+}$and $\mathrm{Be}^{2+}$ in their ground states. In order to put things in perspective, here and in all other tables, three sets of calculations have been performed at each $r_{c}$, namely, (i) exchange-only (ii) XC with Wigner correlation (iii) XC with LYP correlation. Throughout the article, these three results are denoted by labels X-only, XC-Wigner and XC-LYP respectively. This can give us an idea how X-only and HF results compare and contrast. Moreover, it will help us in getting a sense of correlation contribution in current context, approximated by two functionals. In all three occasions (i)-(iii), $S_{\mathrm{r}}$ 's increase with rise in $r_{c}$ and finally merge to corresponding free atom entropy at a sufficiently large $r_{c}$. The reported values of $S_{\mathrm{r}}$ in this table further reinforces the previous conclusions [64,72] that impenetrable walls impose confinements in a way that localizes the electron density, and consequently $S_{\mathbf{r}} \rightarrow-\infty$ when $r_{c} \rightarrow 0$. However, it is important to point out that, in all these cases total energy monotonically decreases with $r_{c}$ eventually reaching the free-atom limit. Actually, with reduction in $r_{c}$ the $r$-space electron density gets compressed and as a consequence, $S_{\mathbf{r}}$ decreases. On the contrary, $S_{\mathrm{p}}$ gradually abates with progress in $r_{c}$. At all $r_{c}$ 's, however, $S_{t}$ maintains the lower bound (6.434) governed by the well-known BBM inequality [62]. The qualitative behavior of $S_{\mathbf{r}}, S_{\mathbf{p}}, S_{t}$ with growth in $r_{c}$ does not change much with atomic charge (Z), although their numerical values differ. In fact, at a given $r_{c}, S_{\mathbf{r}}$ regresses and $S_{\mathrm{p}}$ progresses with advancement of $Z$. With rise in $Z$, electron density gets compressed and hence such a pattern is noticed. Interestingly, while in one-electron systems $S_{t}$ does not depend on $Z$, in a many-electron atom, with change of $Z$ it varies [90]. Earlier $S_{t}$ has been mentioned as a measure of correlation in free systems. Our work establishes the same fact in confinement as well. It is noticed that, for all the three species, $S^{\prime}$ s are identical at very low $r_{c}$ region $(\leq 0.5)$, without or with (either Wigner or LYP) correlation. Furthermore, these two results begin to differ at larger $r_{c}$ indicating correlation effects to assume more significance in the respective free-atom case. In other words, this implies that, at smaller $r_{c}$ region, XC effect is minimum, which enhances with rise in $r_{c}$. Similar conclusions have been found in the energy analysis of confined He in [55]. For He and $\mathrm{Li}^{+}$, these have been estimated by BLYP calculation [64] in most of the $r_{c}$ 's considered here, which are appropriately quoted. Note that the HF values [91] for $S_{\mathbf{r}}, S_{\mathbf{p}}, S_{t}$ in ground state of unconfined $\mathrm{He}$ match reasonably well with our $\mathrm{X}$-only results. Since we are unable to find reference theoretical results for $X$-only $S^{\prime}$ s in the hard confinement, for direct comparison, as a matter of check, a couple of comparisons on respective free systems is provided here. Thus the literature $S_{\mathbf{r}}, S_{\mathbf{p}}, S_{t}$ values of $\mathrm{He}$ and $\mathrm{Li}^{+}$within HF method [91,92], employing $N$-normalized densities, given in footnote of the table, are in reasonable agreement with our X-only values. Recently, in a penetrable confinement calculation within HF, some results on $S_{\mathbf{r}}$ have been presented [72]. The same has also been calculated from a DFT-based study with hybrid exchange functional [52]. Results for He in free-limit from both these studies, presented in footnote show quite decent agreement with ours. It may be noted that in these two aforementioned references $S_{r}$ in $U_{0} \rightarrow \infty$ corresponds to the impenetrable confinement. Highly accurate benchmark-quality result for $S_{\mathbf{r}}$ was calculated from a Hylleraas-type variational method producing a value of 2.7051028 and 1.2552726 for 
free $\mathrm{He}$ and $\mathrm{Li}^{+}$[93] respectively. Correlated results obtained from variational Monte carlo and diffusion Monte Carlo methods [92] for $\mathrm{He}$ and $\mathrm{Li}^{+}$are also cited in the footnote. The current single-determinantal approach quite nicely compares with reference values in the table-XC-LYP providing a slight edge over XC-Wigner. It would be worthwhile to make a comparative energy analysis of these two functionals, which we intend to do in near future. No reference entropies are available for $\mathrm{Be}^{2+}$.

A careful examination of Table 1 reveals that, X-only, XC-Wigner and XC-LYP results provide similar qualitative trend with respect to changes in $r_{c}$, for all three species. Hence in Figure $1, \mathrm{X}$-only $S_{\mathbf{r}}$ $S_{\mathbf{p}}$ and $S_{t}$ are plotted for ground state of all three isoelectronic members, as functions of $r_{c}$ in panels (a)-(c). The first two panels imply that, for a fixed $Z, S_{\mathbf{r}}, S_{\mathbf{p}}$ go up and down respectively with rise in $r_{c}$. On the contrary, for a given $r_{c}$, variation of these two quantities with $Z$ shows opposite trend; the former decays and latter develops as $Z$ advances. These results reinforce the inferences drawn from Table 1. Another point to be noted here is that, with lowering in $r_{c}$ the difference between $S_{\mathbf{r}}$ and $S_{\mathbf{p}}$ corresponding to two successive members of the isoelectronic series, diminishes; in other words, as $r_{c}$ enhances, so does the difference. As $r_{c}$ declines, both average electron-nucleus and electron-electron distances fall down. In stronger confinement regime, the effect of $Z$ on ground state gets dominated by confining potential, resulting in the fact that the three $S_{\mathbf{r}}, S_{\mathbf{p}}$ plots very nearly coincide. For a fixed $Z$, variation of $S_{t}$ with $r_{c}$ in panel (c) suggests that the entropy sum reduces dramatically from its free atomic value as $r_{c}$ is lowered. With enhanced confinement, a distinct minimum followed by a maximum shows up in the curve for all He-like ions. The minimum tends to shift towards left as $Z$ progresses. This observed pattern is in consonance with that found in [64]. 
Table 1. $S_{\mathbf{r}}, S_{\mathbf{p}}, S_{t}$ (a.u.) in the ground states of confined $\mathrm{He}, \mathrm{Li}^{+}$, and $\mathrm{Be}^{2+}$. See the text for details.

\begin{tabular}{|c|c|c|c|c|c|c|c|c|c|c|c|c|c|}
\hline \multirow{2}{*}{ Species } & \multirow{2}{*}{$r_{c}$} & \multicolumn{3}{|c|}{ X-Only } & \multicolumn{3}{|c|}{ XC-Wigner } & \multicolumn{3}{|c|}{ XC-LYP } & \multirow{2}{*}{$\begin{array}{c}\text { Literature }^{1} \\
\mathrm{~S}_{\mathrm{r}}\end{array}$} & \multirow[b]{2}{*}{$S_{p}$} & \multirow[b]{2}{*}{$\mathbf{S}_{t}$} \\
\hline & & $S_{r}$ & $S_{p}$ & $\mathbf{S}_{t}$ & $S_{r}$ & $\mathrm{~S}_{\mathrm{p}}$ & $\mathbf{S}_{t}$ & $S_{r}$ & $\mathrm{~S}_{\mathrm{p}}$ & $\mathrm{S}_{t}$ & & & \\
\hline \multirow{11}{*}{$\mathrm{He}^{2,3}$} & 0.1 & -6.2534 & 12.855 & 6.5744 & -6.2534 & 12.855 & 6.5744 & -6.2534 & 12.855 & 6.5744 & & & \\
\hline & 0.3 & -3.004 & 9.580 & 6.576 & -3.004 & 9.580 & 6.576 & -3.004 & 9.580 & 6.576 & -2.988 & 9.488 & 6.5 \\
\hline & 0.5 & -1.525 & 8.075 & 6.550 & -1.525 & 8.075 & 6.550 & -1.525 & 8.075 & 6.550 & -1.498 & 8.023 & 6.525 \\
\hline & 1 & 0.389 & 6.118 & 6.507 & 0.387 & 6.119 & 6.506 & 0.388 & 6.118 & 6.506 & 0.4326 & 6.078 & 6.51 \\
\hline & 1.4 & 1.22 & 5.273 & 6.493 & 1.22 & 5.276 & 6.49 & 1.22 & 5.327 & 6.547 & 1.2739 & 5.234 & 6.508 \\
\hline & 2 & 1.97 & 4.547 & 6.517 & 1.96 & 4.555 & 6.515 & 1.96 & 4.549 & 6.509 & 2.0097 & 4.519 & 6.528 \\
\hline & 3 & 2.50 & 4.061 & 6.561 & 2.48 & 4.080 & 6.560 & 2.50 & 4.068 & 6.568 & 2.5241 & 4.057 & 6.581 \\
\hline & 4 & 2.65 & 3.943 & 6.593 & 2.63 & 3.969 & 6.599 & 2.64 & 3.952 & 6.592 & 2.6651 & 3.945 & 6.61 \\
\hline & 5 & 2.68 & 3.921 & 6.608 & 2.65 & 3.95 & 6.60 & 2.67 & 3.932 & 6.602 & 2.7042 & 3.918 & 6.622 \\
\hline & 6 & 2.69 & 3.918 & 6.608 & 2.66 & 3.95 & 6.61 & 2.68 & 3.93 & 6.61 & 2.7106 & 3.914 & 6.625 \\
\hline & 7 & 2.69 & 3.918 & 6.608 & 2.66 & 3.95 & 6.61 & 2.68 & 3.93 & 6.61 & 2.7117 & 3.913 & 6.625 \\
\hline \multirow{9}{*}{$\mathrm{Li}^{+4,5}$} & 0.1 & -6.2665 & 12.860 & 6.5935 & -6.2665 & 12.860 & 6.5935 & -6.2665 & 12.860 & 6.5935 & & & \\
\hline & 0.3 & -3.050 & 9.604 & 6.554 & -3.050 & 9.604 & 6.554 & -3.050 & 9.604 & 6.554 & -3.034 & 9.538 & 6.504 \\
\hline & 0.8 & -0.392 & 6.890 & 6.498 & -0.393 & 6.891 & 6.498 & -0.392 & 6.890 & 6.498 & -0.353 & 6.849 & 6.496 \\
\hline & 1 & 0.12 & 6.376 & 6.496 & 0.121 & 6.378 & 6.499 & 0.122 & 6.377 & 6.499 & 0.1659 & 6.335 & 6.501 \\
\hline & 2 & 1.135 & 5.431 & 6.566 & 1.12 & 5.440 & 6.56 & 1.13 & 5.43 & 6.56 & 1.174 & 5.4 & 6.574 \\
\hline & 2.5 & 1.22 & 5.361 & 6.581 & 1.21 & 5.373 & 6.58 & 1.22 & 5.36 & 6.58 & 1.2618 & 5.331 & 6.593 \\
\hline & 3 & 1.24 & 5.346 & 6.586 & 1.23 & 5.358 & 6.58 & 1.24 & 5.34 & 6.58 & 1.2878 & 5.313 & 6.601 \\
\hline & 4 & 1.25 & 5.343 & 6.593 & 1.23 & 5.355 & 6.58 & 1.24 & 5.34 & 6.58 & 1.2942 & 5.309 & 6.603 \\
\hline & 7 & 1.25 & 5.343 & 6.593 & 1.23 & 5.355 & 6.58 & 1.24 & 5.34 & 6.58 & & & \\
\hline \multirow{9}{*}{$\mathrm{Be}^{2+}$} & 0.1 & -6.2801 & 12.866 & 6.5859 & -6.2801 & 12.866 & 6.5859 & -6.2801 & 12.866 & 6.5859 & & & \\
\hline & 0.3 & -3.102 & 9.636 & 6.534 & -3.102 & 9.636 & 6.534 & -3.102 & 9.636 & 6.534 & & & \\
\hline & 0.5 & -1.725 & 8.226 & 6.501 & -1.725 & 8.226 & 6.501 & -1.725 & 8.226 & 6.501 & & & \\
\hline & 1 & -0.229 & 6.748 & 6.519 & -0.231 & 6.750 & 6.519 & -0.229 & 6.748 & 6.519 & & & \\
\hline & 1.5 & 0.191 & 6.373 & 6.564 & 0.186 & 6.378 & 6.564 & 0.190 & 6.374 & 6.564 & & & \\
\hline & 2 & 0.27 & 6.311 & 6.581 & 0.26 & 6.317 & 6.577 & 0.26 & 6.312 & 6.572 & & & \\
\hline & 2.5 & 0.28 & 6.305 & 6.585 & 0.27 & 6.311 & 6.581 & 0.26 & 6.306 & 6.566 & & & \\
\hline & 3 & 0.28 & 6.305 & 6.585 & 0.27 & 6.311 & 6.581 & 0.26 & 6.306 & 6.566 & & & \\
\hline & 7 & 0.28 & 6.305 & 6.585 & 0.27 & 6.311 & 6.581 & 0.26 & 6.306 & 6.566 & & & \\
\hline
\end{tabular}

${ }^{1}$ DFT calculation [64], using the BLYP functional. ${ }^{2}$ For the free atom, using $N$-normalized HF density; $S_{\mathbf{r}}: 4.01$ [72], 4.06 [91], 4.0100 [92], 4.0107 [52]; $S_{\mathbf{p}}: 6.45$ [91]; $S_{t}: 10.52$ [91]. Our exchange-only (X-only) results are 4.00, 6.45, and 10.52 respectively. ${ }^{3}$ Correlated $S_{\mathrm{r}}$ values in the free He atom: (i) unit-normalized density: 2.7051028 [93], 2.705 [94]; (ii) N-normalized density: 4.40106; (variational Monte Carlo), 4.0256 (diffusion Monte Carlo) [92]. Our exchange-correlation (XC) results are 3.93 (XC-Wigner) and 3.96 (XC-Lee-Yang-Parr (LYP)). ${ }^{4} \mathrm{HF} S_{\mathrm{r}}$ value in the free $\mathrm{Li}^{+}$ion using $\mathrm{N}$-normalized density: 1.1023 [92]. Our X-only result is $1.11 .{ }^{5}$ Correlated $S_{\mathrm{r}}$ value in the $\mathrm{Li}^{+}$ion: (i) unit-normalized density: 1.2552726 [93]; (ii) N-normalized density: 1.1204 (Variational Monte Carlo), [92]. 1.1143 (diffusion Monte Carlo). Our XC results are: 1.08 (XC-Wigner), 1.10 (XC-LYP). 
Now, we move on to some low-lying singly excited state- $S_{\mathbf{r}}, S_{\mathbf{p}}, S_{t}$ for $\mathrm{He}, \mathrm{Li}^{+}$and $\mathrm{Be}^{2+}$ in Tables 2-4. Following the presentation strategy of previous table, it reports results for $1 \mathrm{~s} 2 \mathrm{~s}^{3} \mathrm{~S}, 1 \mathrm{~s} 2 \mathrm{p}^{3} \mathrm{P}$ and $1 \mathrm{~s} 3 \mathrm{~d}^{3} \mathrm{D}$ states respectively. Like the ground state, here also in all three states, both X-only and correlation-included $S_{\mathbf{r}}, S_{\mathbf{p}}$ moves up and down respectively with growth in $r_{c}$. In all cases again $S_{t}$ obeys the stipulated lower bound [62]. The qualitative pattern of $S_{\mathbf{r}}, S_{\mathbf{p}}, S_{t}$ with progress in $r_{c}$ remains invariant with change of $Z$. However, their numerical values alter substantially. Similar to the ground state, at a fixed $r_{c}, S_{\mathbf{r}}$ falls off and $S_{\mathbf{p}}$ enhances with advancement of $Z$. Once again, for a given $Z$, at low $r_{c}$ region, $\mathrm{X}$-only, XC-Wigner and XC-LYP results practically merge with each other, signifying that the effect of correlation is somewhat less impactful in stronger confinement region, as the confining potential leads the contribution in this scenario. Whereas, with rise in $r_{c}$, the correlation effect prevails, indicating its importance in free conditions. Except the free atom-limit of $S_{\mathrm{r}}$ in $1 \mathrm{~s} 2 \mathrm{~s}{ }^{3} \mathrm{~S} \mathrm{He}$, no reference results could be found for any of the confined states and we hope the present work would provide useful guideline in future.

Table 2. $S_{\mathbf{r}}, S_{\mathbf{p}}, S_{t}$ (a.u.) in $1 \mathrm{~s} 2 \mathrm{~s}^{3} \mathrm{~S}$ states of confined $\mathrm{He}, \mathrm{Li}^{+}$, and $\mathrm{Be}^{2+}$. See the text for details.

\begin{tabular}{|c|c|c|c|c|c|c|c|c|c|c|}
\hline \multirow{2}{*}{ Species } & \multirow{2}{*}{$r_{c}$} & \multicolumn{3}{|c|}{ X-Only } & \multicolumn{3}{|c|}{ XC-Wigner } & \multicolumn{3}{|c|}{ XC-LYP } \\
\hline & & $S_{r}$ & $S_{p}$ & $\mathrm{~S}_{t}$ & $S_{r}$ & $S_{p}$ & $\mathrm{~S}_{t}$ & $S_{r}$ & $S_{p}$ & $\mathrm{~S}_{t}$ \\
\hline \multirow{10}{*}{$\mathrm{He}^{1}$} & 0.1 & -6.2172 & 14.190 & 7.9728 & -6.2172 & 14.190 & 7.9728 & -6.2172 & 14.190 & 7.9728 \\
\hline & 0.5 & -1.4472 & 9.376 & 7.9288 & -1.4472 & 9.376 & 7.9288 & -1.4472 & 9.3756 & 7.9288 \\
\hline & 1 & 0.547 & 7.333 & 7.880 & 0.547 & 7.333 & 7.880 & 0.547 & 7.333 & 7.880 \\
\hline & 2 & 2.417 & 5.409 & 7.826 & 2.415 & 5.410 & 7.825 & 2.417 & 5.409 & 7.826 \\
\hline & 4 & 3.956 & 3.86 & 7.819 & 3.948 & 3.87 & 7.818 & 3.953 & 3.86 & 7.813 \\
\hline & 6 & 4.60 & 3.22 & 7.82 & 4.59 & 3.23 & 7.82 & 4.59 & 3.23 & 7.82 \\
\hline & 6.5 & 4.71 & 3.11 & 7.82 & 4.69 & 3.13 & 7.82 & 4.69 & 3.13 & 7.82 \\
\hline & 7.5 & 4.87 & 2.94 & 7.81 & 4.85 & 2.96 & 7.81 & 4.85 & 2.97 & 7.82 \\
\hline & 8.5 & 4.99 & 2.81 & 7.80 & 4.96 & 2.84 & 7.80 & 4.96 & 2.85 & 7.81 \\
\hline & 10 & 5.10 & 2.69 & 7.79 & 5.06 & 2.72 & 7.78 & 5.03 & 2.75 & 7.78 \\
\hline \multirow{10}{*}{$\mathrm{Li}^{+}$} & 0.1 & -6.2246 & 14.191 & 7.9664 & -6.2246 & 14.191 & 7.9664 & -6.2246 & 14.191 & 7.9664 \\
\hline & 0.5 & -1.4905 & 9.392 & 7.9015 & -1.4905 & 9.392 & 7.9015 & -1.4905 & 9.392 & 7.9015 \\
\hline & 1 & 0.442 & 7.401 & 7.843 & 0.442 & 7.401 & 7.843 & 0.442 & 7.401 & 7.843 \\
\hline & 1.5 & 1.481 & 6.335 & 7.816 & 1.480 & 6.336 & 7.816 & 1.481 & 6.336 & 7.817 \\
\hline & 2 & 2.141 & 5.669 & 7.810 & 2.138 & 5.671 & 7.809 & 2.140 & 5.669 & 7.809 \\
\hline & 3 & 2.910 & 4.90 & 7.810 & 2.905 & 4.90 & 7.805 & 2.909 & 4.905 & 7.814 \\
\hline & 4 & 3.32 & 4.49 & 7.81 & 3.312 & 4.49 & 7.802 & 3.31 & 4.49 & 7.80 \\
\hline & 7 & 3.70 & 4.07 & 7.77 & 3.68 & 4.09 & 7.77 & 3.69 & 4.09 & 7.78 \\
\hline & 8.5 & 3.72 & 4.05 & 7.77 & 3.70 & 4.07 & 7.77 & 3.70 & 4.07 & 7.77 \\
\hline & 10 & 3.72 & 4.05 & 7.77 & 3.70 & 4.07 & 7.77 & 3.70 & 4.07 & 7.77 \\
\hline \multirow{9}{*}{$\mathrm{Be}^{2+}$} & 0.1 & -6.2320 & 14.191 & 7.9590 & -6.2320 & 14.191 & 7.9590 & -6.2320 & 14.191 & 7.9590 \\
\hline & 0.5 & -1.5376 & 9.415 & 7.8774 & -1.537 & 9.415 & 7.8774 & -1.5376 & 9.415 & 7.8774 \\
\hline & 1 & 0.322 & 7.499 & 7.821 & 0.322 & 7.500 & 7.822 & 0.322 & 7.499 & 7.821 \\
\hline & 2 & 1.829 & 5.981 & 7.810 & 1.826 & 5.983 & 7.809 & 1.828 & 5.981 & 7.809 \\
\hline & 3 & 2.42 & 5.38 & 7.80 & 2.141 & 5.39 & 7.531 & 2.418 & 5.38 & 7.798 \\
\hline & 4 & 2.66 & 5.12 & 7.78 & 2.651 & 5.13 & 7.781 & 2.656 & 5.12 & 7.776 \\
\hline & 5 & 2.73 & 5.03 & 7.76 & 2.72 & 5.05 & 7.77 & 2.72 & 5.04 & 7.76 \\
\hline & 6 & 2.75 & 5.02 & 7.77 & 2.73 & 5.03 & 7.76 & 2.74 & 5.02 & 7.76 \\
\hline & 20 & 2.75 & 5.02 & 7.77 & 2.73 & 5.03 & 7.76 & 2.74 & 5.02 & 7.76 \\
\hline
\end{tabular}

${ }^{1}$ Correlated $S_{\mathbf{r}}$ value in the free atom is: 5.239 [94]. 

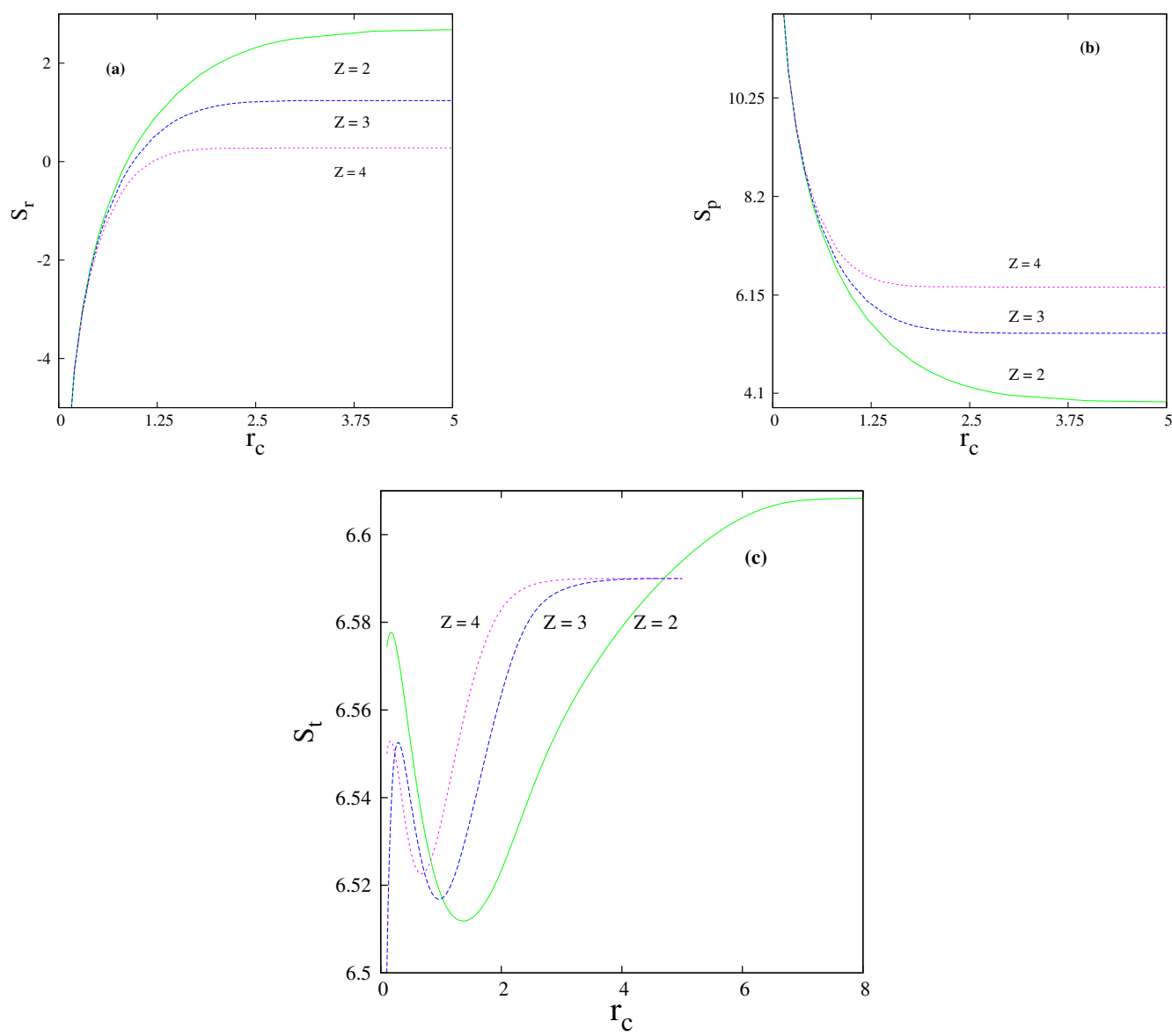

Figure 1. Variation of $S_{\mathbf{r}}, S_{\mathbf{p}}, S_{t}$ for He-isoelectronic series $(Z=2-4)$ with $r_{c}$ in $(\mathbf{a}-\mathbf{c})$.

In order to gain a better understanding of the effect of confinement on excited states, $S_{\mathbf{r}}, S_{\mathbf{p}}$ in compressed He have been plotted in panels (a), (b) of Figure 2, for three triplet singly excited states arising from configuration $1 \mathrm{sns}$, corresponding to $n=2-4$. Since correlation does not affect the results qualitatively, for this purpose, it suffices to consider X-only results. With this in mind, here and in next figure (Figure 3), only X-only entropies are shown. It is obvious from these plots that, for excited states, as in Figure 1, $S_{\mathbf{r}}$ gains with rise in $r_{c}$, while $S_{\mathbf{p}}$ declines. Now for a fixed $r_{c}$, the behavior of $S_{\mathbf{r}}$ with $n$ (in this series), shows interesting pattern. For a large enough value of $r_{c}$, which corresponds to the free-atom limit of $\mathrm{He}$ of the state under consideration, $S_{\mathbf{r}}$ progresses as $n$ grows. Though it may not be so apparent from the data presented in respective table or plot, as the maximum range of $r_{c}$ presented here is 10 a.u. This can be concluded from the fact that $S_{\mathrm{r}}$ for 1s2s, $1 \mathrm{~s} 3 \mathrm{~s}$ and $1 \mathrm{~s} 4 \mathrm{~s}$ triplet states in the free limit are 5.20,6.53, 7.43 respectively, signifying a progressive delocalization. But this pattern gets dissolved with reduction in $r_{c}$ and crossing between $S_{\mathbf{r}}$ for different states occurs. From the inset plots of panel (a) it is noticed that, at $r_{c} \approx 7.43$ there is a crossing between $S_{\mathbf{r}}^{(1 s 2 s)}$ and $S_{\mathbf{r}}^{(1 s 4 s)}$; another crossing occurs at $r_{c} \approx 6.36$ between $S_{\mathbf{r}}^{(1 s 3 s)}$ and $S_{\mathbf{r}}^{(1 s 4 s)}$. With further gain in confinement strength, at $r_{c} \approx 0.33$ and 0.34 , crossings take place between $S_{\mathbf{r}}^{(1 s 2 s)}, S_{\mathbf{r}}^{(1 s 4 s)}$ and $S_{\mathbf{r}}^{(1 s 2 s)}, S_{\mathbf{r}}^{(1 s 3 s)}$ respectively. Hence, one encounters frequent change in the order arrangement on proceeding from free to strong confined regime. In the strong confinement regime $\left(r_{c} \approx 0.1\right)$ the following ordering of entropy holds good: $S_{\mathbf{r}}^{(1 s 2 s)}>S_{\mathbf{r}}^{(1 s 3 s)}>S_{\mathbf{r}}^{(1 s 4 s)}$. Apparently, there exists an interplay between two competing effects, namely, (i) radial confinement (localization) and (ii) accumulation of nodes and humps with growth in $n$ (delocalization). And these two opposing forces control the ordering of $S$ values of these states. Similarly, in $p$ space also, such crossovers prevail at various $r_{c}$ 's. However, at $r_{c} \rightarrow \infty$ limit and $r_{c}=0.1$, $S_{\mathbf{p}}$ shows opposite trend to that of $S_{\mathbf{r}}$. 
Table 3. $S_{\mathbf{r}}, S_{\mathbf{p}}, S_{t}$ (a.u.) in $1 \mathrm{~s} 2 \mathrm{p}{ }^{3} \mathrm{P}$ states of confined $\mathrm{He}, \mathrm{Li}^{+}$, and $\mathrm{Be}^{2+}$. See the text for details.

\begin{tabular}{|c|c|c|c|c|c|c|c|c|c|c|}
\hline \multirow{2}{*}{ Species } & \multirow{2}{*}{$r_{c}$} & \multicolumn{3}{|c|}{ X-Only } & \multicolumn{3}{|c|}{ XC-Wigner } & \multicolumn{3}{|c|}{ XC-LYP } \\
\hline & & $\mathrm{S}_{\mathbf{r}}$ & $S_{p}$ & $\mathbf{S}_{t}$ & $S_{r}$ & $S_{p}$ & $\mathbf{S}_{t}$ & $S_{r}$ & $S_{p}$ & $\mathbf{S}_{t}$ \\
\hline \multirow{10}{*}{$\mathrm{He}^{1}$} & 0.5 & -1.392 & 8.596 & 7.204 & -1.392 & 8.596 & 7.204 & -1.392 & 8.596 & 7.204 \\
\hline & 0.8 & -0.031 & 7.213 & 7.182 & -0.031 & 7.213 & 7.182 & -0.031 & 7.213 & 7.182 \\
\hline & 1 & 0.60 & 6.570 & 7.170 & 0.60 & 6.570 & 7.170 & 0.60 & 6.570 & 7.170 \\
\hline & 3 & 3.26 & 4.04 & 7.30 & 3.26 & 4.05 & 7.31 & 3.26 & 4.04 & 7.30 \\
\hline & 5 & 4.09 & 3.49 & 7.58 & 4.08 & 3.50 & 7.58 & 4.08 & 3.50 & 7.58 \\
\hline & 6 & 4.35 & 3.34 & 7.69 & 4.32 & 3.36 & 7.68 & 4.33 & 3.35 & 7.68 \\
\hline & 7 & 4.54 & 3.23 & 7.77 & 4.51 & 3.25 & 7.76 & 4.51 & 3.25 & 7.76 \\
\hline & 7.6 & 4.63 & 3.18 & 7.81 & 4.58 & 3.21 & 7.79 & 4.58 & 3.21 & 7.79 \\
\hline & 8 & 4.68 & 3.15 & 7.83 & 4.65 & 3.18 & 7.83 & 4.64 & 3.18 & 7.82 \\
\hline & 10 & 4.86 & 3.04 & 7.90 & 4.82 & 3.08 & 7.90 & 4.78 & 3.10 & 7.88 \\
\hline \multirow{10}{*}{$\mathrm{Li}^{+}$} & 0.5 & -1.437 & 8.620 & 7.183 & -1.437 & 8.620 & 7.183 & -1.437 & 8.620 & 7.183 \\
\hline & 0.8 & -0.121 & 7.284 & 7.163 & -0.121 & 7.284 & 7.163 & -0.121 & 7.284 & 7.163 \\
\hline & 1 & 0.471 & 6.693 & 7.164 & 0.470 & 6.694 & 7.164 & 0.47 & 6.69 & 7.16 \\
\hline & 1.5 & 1.44 & 5.91 & 7.35 & 1.438 & 5.91 & 7.358 & 1.44 & 5.91 & 7.35 \\
\hline & 2 & 2.009 & 5.29 & 7.299 & 2.005 & 5.30 & 7.305 & 2.008 & 5.30 & 7.308 \\
\hline & 3 & 2.65 & 4.85 & 7.50 & 2.64 & 4.86 & 7.50 & 2.653 & 4.86 & 7.513 \\
\hline & 6 & 3.33 & 4.47 & 7.80 & 3.31 & 4.49 & 7.80 & 3.31 & 4.48 & 7.79 \\
\hline & 8 & 3.39 & 4.45 & 7.84 & 3.36 & 4.47 & 7.83 & 3.37 & 4.47 & 7.84 \\
\hline & 9 & 3.40 & 4.45 & 7.85 & 3.36 & 4.47 & 7.83 & 3.37 & 4.47 & 7.84 \\
\hline & 10 & 3.40 & 4.45 & 7.85 & 3.37 & 4.47 & 7.84 & 3.37 & 4.47 & 7.84 \\
\hline \multirow{8}{*}{$\mathrm{Be}^{2+}$} & 0.5 & -1.490 & 8.658 & 7.168 & -1.490 & 8.658 & 7.168 & -1.490 & 8.658 & 7.168 \\
\hline & 0.8 & -0.235 & 7.402 & 7.167 & -0.235 & 7.402 & 7.167 & -0.235 & 7.402 & 7.167 \\
\hline & 1.2 & 0.698 & 6.532 & 7.23 & 0.697 & 6.534 & 7.231 & 0.698 & 6.532 & 7.23 \\
\hline & 2 & 1.589 & 5.857 & 7.446 & 1.585 & 5.861 & 7.446 & 1.588 & 5.858 & 7.446 \\
\hline & 2.5 & 1.892 & 5.670 & 7.562 & 1.88 & 5.67 & 7.55 & 1.890 & 5.701 & 7.591 \\
\hline & 5 & 2.37 & 5.41 & 7.78 & 2.35 & 5.43 & 7.78 & 2.36 & 5.42 & 7.78 \\
\hline & 6 & 2.38 & 5.41 & 7.79 & 2.36 & 5.42 & 7.78 & 2.37 & 5.42 & 7.79 \\
\hline & 10 & 2.38 & 5.41 & 7.79 & 2.36 & 5.42 & 7.78 & 2.37 & 5.42 & 7.79 \\
\hline
\end{tabular}

${ }^{1}$ Correlated $S_{\mathrm{r}}$ value in the free atom is: 5.356 [74].
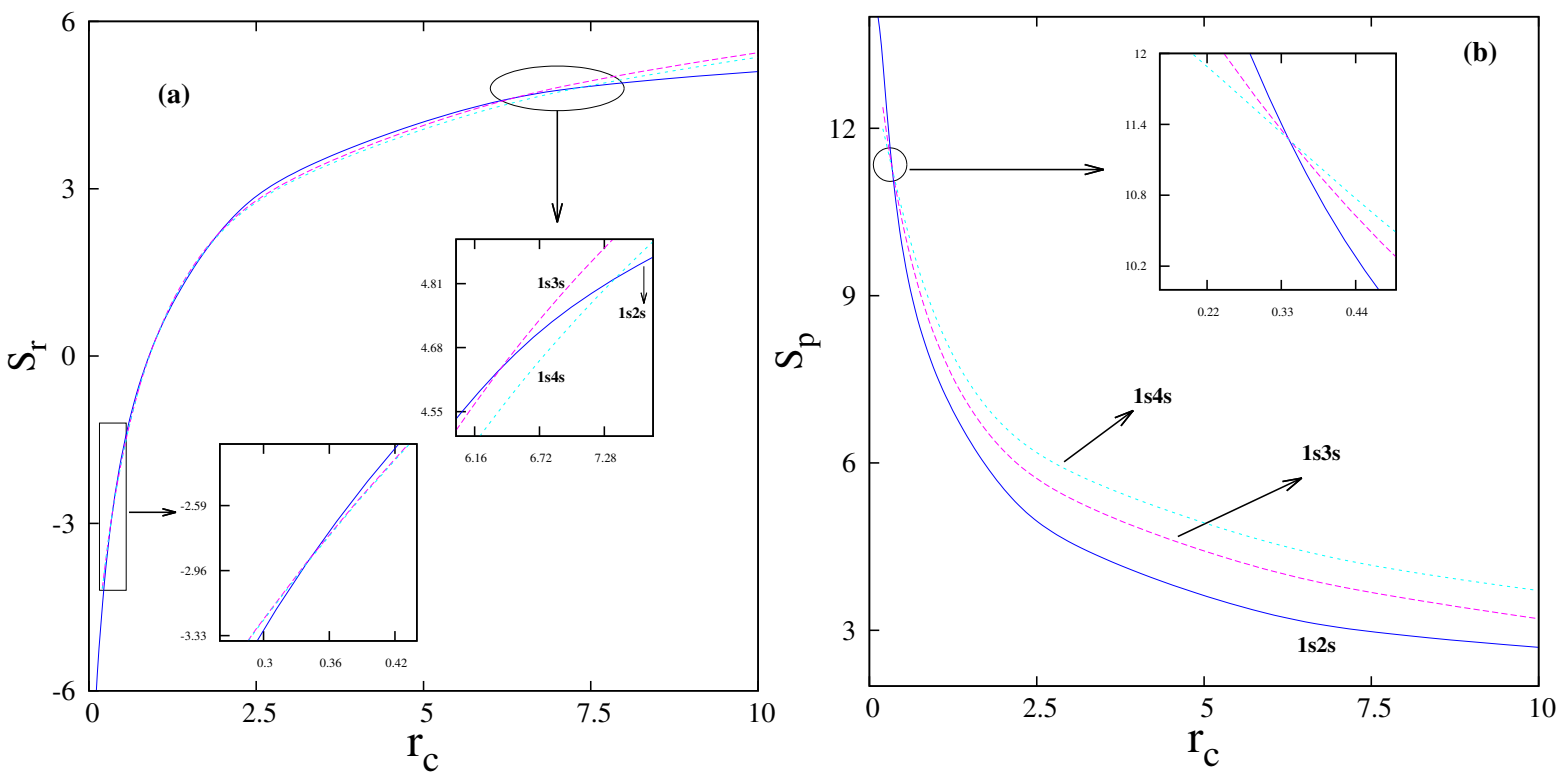

Figure 2. Variation of $S_{\mathbf{r}}, S_{\mathbf{p}}$ in $1 \mathrm{sns}{ }^{3} \mathrm{~S}(n=2-4)$ states of confined He with $r_{c}$. See the text for details. 
Table 4. $S_{\mathbf{r}}, S_{\mathbf{p}}, S_{t}$ (a.u.) in $1 \mathrm{~s} 3 \mathrm{~d}^{3} \mathrm{D}$ states of confined $\mathrm{He}, \mathrm{Li}^{+}$, and $\mathrm{Be}^{2+}$. See the text for details.

\begin{tabular}{|c|c|c|c|c|c|c|c|c|c|c|}
\hline \multirow{2}{*}{ Species } & \multirow{2}{*}{$r_{c}$} & \multicolumn{3}{|c|}{ X-only } & \multicolumn{3}{|c|}{ XC-Wigner } & \multicolumn{3}{|c|}{ XC-LYP } \\
\hline & & $\mathrm{S}_{\mathrm{r}}$ & $S_{p}$ & $\mathbf{S}_{t}$ & $S_{\mathbf{r}}$ & $s_{p}$ & $\mathrm{~S}_{t}$ & $S_{\mathbf{r}}$ & $S_{p}$ & $\mathbf{S}_{t}$ \\
\hline \multirow{10}{*}{$\mathrm{He}^{1}$} & 0.5 & -1.3053 & 9.110 & 7.8047 & -1.3053 & 9.110 & 7.8047 & -1.3053 & 9.110 & 7.8047 \\
\hline & 1 & 0.707 & 7.073 & 7.780 & 0.706 & 7.073 & 7.779 & 0.707 & 7.073 & 7.780 \\
\hline & 1.5 & 1.828 & 5.938 & 7.766 & 1.826 & 5.939 & 7.765 & 1.827 & 5.938 & 7.765 \\
\hline & 2.6 & 3.155 & 4.640 & 7.795 & 3.150 & 4.645 & 7.795 & 3.153 & 4.641 & 7.794 \\
\hline & 4 & 3.960 & 3.957 & 7.917 & 3.951 & 3.967 & 7.918 & 3.957 & 3.960 & 7.917 \\
\hline & 5 & 4.327 & 3.687 & 8.014 & 4.317 & 3.699 & 8.016 & 4.323 & 3.691 & 8.014 \\
\hline & 6 & 4.61 & 3.48 & 8.09 & 4.60 & 3.50 & 8.10 & 4.60 & 3.49 & 8.09 \\
\hline & 7 & 4.85 & 3.33 & 8.18 & 4.83 & 3.34 & 8.17 & 4.83 & 3.34 & 8.17 \\
\hline & 8 & 5.04 & 3.19 & 8.23 & 5.03 & 3.21 & 8.24 & 5.02 & 3.22 & 8.24 \\
\hline & 10 & 5.36 & 2.97 & 8.33 & 5.35 & 2.99 & 8.34 & 5.27 & 3.04 & 8.24 \\
\hline \multirow{11}{*}{$\mathrm{Li}^{+}$} & 0.5 & -1.339 & 9.129 & 7.790 & -1.339 & 9.129 & 7.790 & -1.339 & 9.129 & 7.790 \\
\hline & 0.8 & -0.003 & 7.774 & 7.771 & -0.003 & 7.774 & 7.771 & -0.003 & 7.774 & 7.771 \\
\hline & 1 & 0.601 & 7.161 & 7.762 & 0.601 & 7.161 & 7.762 & 0.601 & 7.161 & 7.762 \\
\hline & 2.5 & 2.611 & 5.27 & 7.881 & 2.608 & 5.280 & 7.888 & 2.611 & 5.277 & 7.888 \\
\hline & 3 & 2.918 & 5.042 & 7.960 & 2.914 & 5.047 & 7.961 & 2.917 & 5.043 & 7.960 \\
\hline & 4 & 3.373 & 4.72 & 8.093 & 3.36 & 4.73 & 8.09 & 3.37 & 4.72 & 8.09 \\
\hline & 5 & 3.704 & 4.50 & 8.204 & 3.69 & 4.51 & 8.20 & 3.70 & 4.50 & 8.20 \\
\hline & 6.5 & 4.06 & 4.26 & 8.32 & 4.05 & 4.27 & 8.32 & 4.05 & 4.27 & 8.32 \\
\hline & 7 & 4.16 & 4.20 & 8.36 & 4.15 & 4.21 & 8.36 & 4.14 & 4.21 & 8.35 \\
\hline & 7.5 & 4.24 & 4.14 & 8.38 & 4.23 & 4.15 & 8.38 & 4.22 & 4.16 & 8.38 \\
\hline & 10 & 4.53 & 3.97 & 8.50 & 4.51 & 3.99 & 8.50 & 4.46 & 4.03 & 8.49 \\
\hline \multirow{10}{*}{$\mathrm{Be}^{2+}$} & 0.5 & -1.3803 & 9.157 & 7.776 & -1.3803 & 9.157 & 7.776 & -1.3803 & 9.157 & 7.776 \\
\hline & 0.8 & -0.097 & 7.857 & 7.760 & -0.097 & 7.857 & 7.760 & -0.097 & 7.857 & 7.760 \\
\hline & 1 & 0.460 & 7.303 & 7.763 & 0.459 & 7.304 & 7.763 & 0.460 & 7.303 & 7.763 \\
\hline & 2.5 & 2.21 & 5.79 & 8.00 & 2.211 & 5.79 & 8.001 & 2.213 & 5.79 & 8.003 \\
\hline & 3 & 2.496 & 5.59 & 8.086 & 2.492 & 5.60 & 8.092 & 2.495 & 5.59 & 8.085 \\
\hline & 4 & 2.911 & 5.32 & 8.231 & 2.907 & 5.32 & 8.227 & 2.909 & 5.32 & 8.229 \\
\hline & 7 & 3.53 & 4.94 & 8.47 & 3.51 & 4.96 & 8.47 & 3.51 & 4.96 & 8.47 \\
\hline & 8 & 3.60 & 4.91 & 8.51 & 3.59 & 4.92 & 8.51 & 3.58 & 4.93 & 8.51 \\
\hline & 8.5 & 3.63 & 4.90 & 8.53 & 3.61 & 4.92 & 8.53 & 3.60 & 4.92 & 8.52 \\
\hline & 10 & 3.66 & 4.90 & 8.62 & 3.64 & 4.91 & 8.55 & 3.62 & 4.92 & 8.54 \\
\hline
\end{tabular}

${ }^{1}$ Correlated $S_{\mathbf{r}}$ value in the free atom is: 6.634 [74].

As a continuation of the earlier discussion, we present in Figure 3, X-only $S_{\mathbf{r}}, S_{\mathbf{p}}$ and $S_{t}$ as a functions of $r_{c}$ corresponding to ${ }^{3} \mathrm{~S},{ }^{3} \mathrm{P}$ and ${ }^{3} \mathrm{D}$ states resulting from $1 \mathrm{~s} 3 \mathrm{~s}, 1 \mathrm{~s} 3 \mathrm{p}$ and $1 \mathrm{~s} 3 \mathrm{~d}$ configurations of He, in panels (a)-(c). From panel (a), at $r_{c} \approx 10$, we obtain the following ordering of entropy: $S_{\mathbf{r}}\left({ }^{3} S\right)>S_{\mathbf{r}}\left({ }^{3} P\right)>S_{\mathbf{r}}\left({ }^{3} D\right)$, indicating a drop in fluctuation as one passes from ${ }^{3} S$ to ${ }^{3} \mathrm{P}$ to ${ }^{3} \mathrm{D}$. Similar to that in Figure 2, here also, multiple crossovers take place at intermediate and lower $r_{c}$ region and eventually settles with the following sequential order $S_{\mathbf{r}}\left({ }^{3} P\right)>S_{\mathbf{r}}\left({ }^{3} S\right)>S_{\mathbf{r}}\left({ }^{3} D\right)$ at $\left(r_{c} \approx 0.1\right)$, representing strong confinement region. Now in conjugate space, at higher $r_{c}$ region $\approx 20, S_{\mathbf{p}}$ displays an exact opposite trend to that of $S_{\mathrm{r}}$ in the free limit, which is depicted in panel (b). This is obvious as the more localized a state is in $r$ space, the more diffused it is in $p$ space. Here also, due to crossover between states this pattern gets dissolved at lower $r_{c}$, leading to an ordering as $S_{\mathbf{p}}\left({ }^{3} S\right)>S_{\mathbf{r}}\left({ }^{3} P\right)>S_{\mathbf{r}}\left({ }^{3} D\right)$, at $r_{c} \approx 0.1$. Panel (c) portrays the response of $S_{t}$ which verifies that the lower bound is maintained throughout entire confinement region. 

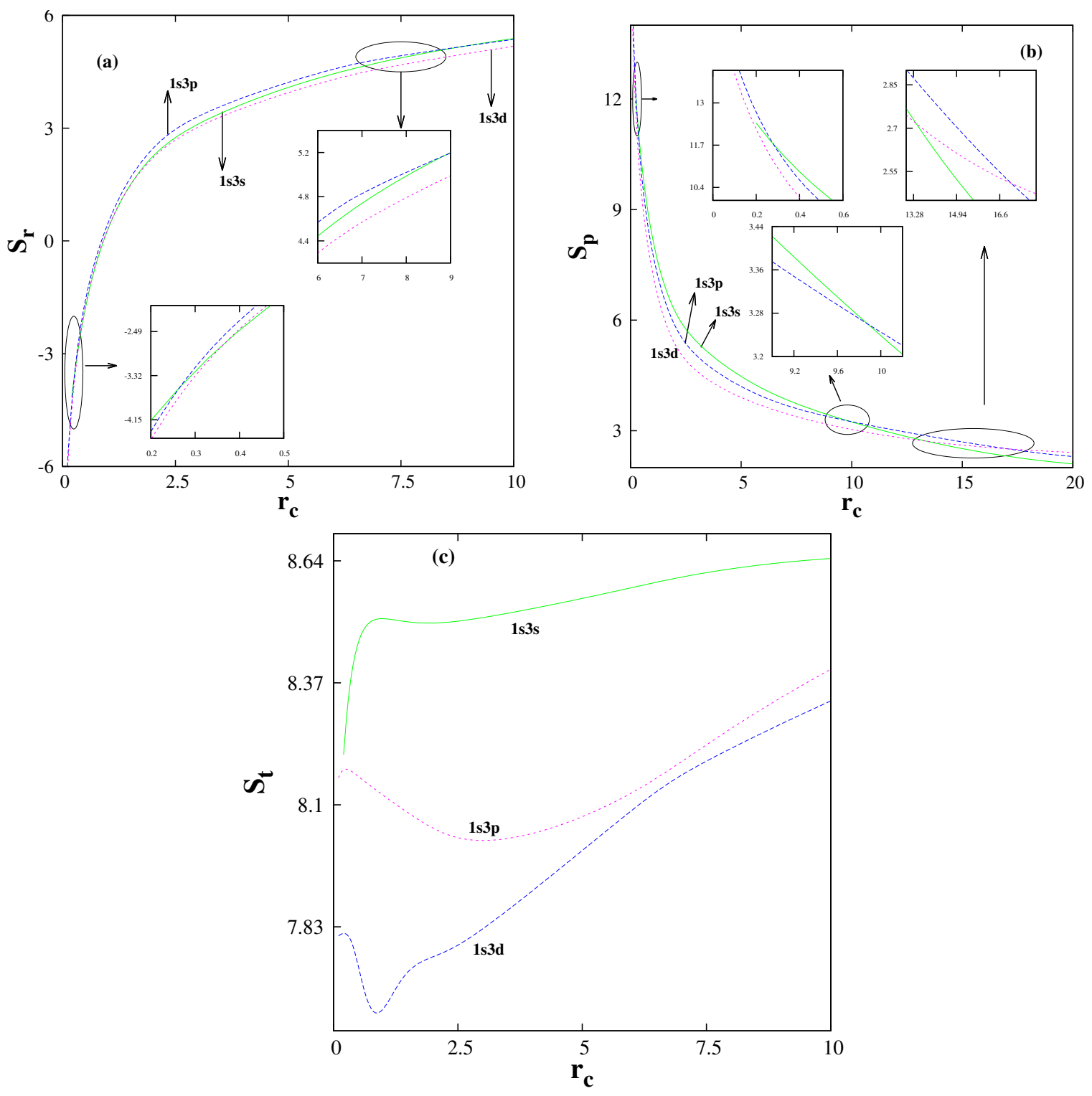

Figure 3. $S_{\mathbf{r}}, S_{\mathbf{p}}, S_{t}$ in $1 \mathrm{~s} 3 \mathrm{~s}^{3} \mathrm{~S}, 1 \mathrm{~s} 3 \mathrm{p}^{3} \mathrm{P}, 1 \mathrm{~s} 3 \mathrm{~d}{ }^{3} \mathrm{D}$ states of confined He, against $r_{c}$. See the text for details.

\section{Conclusions}

Shannon information entropy (in $r$ and $p$ spaces) was analyzed for confined He iso-electronic series. Ground and excited states were studied via a simple DFT method, by solving the radial KS equation through a generalized Legendre pseudospectral method. Some attempts are known for $S$ of the free He atom, as well as its confinement within a soft, penetrable boundary. However, to the best of our knowledge, this is the first such systematic study of information in a confined two-electron atom within a rigid, impenetrable spherical cage. Apart from the ground state, several low-lying singly excited triplet states of the iso-electronic series were considered. As the $\mathrm{X}$-only entropies were comparable to their HF counterparts in the free atom limit, it was expected that this would also hold in the confined case as well. The effects of electron correlation were probed through two correlation functionals. For the states considered here, the correlation contribution remained rather small in the low $r_{c}$ regime, assuming greater significance as the latter approached the free atom limit. It was observed that the two correlation functionals offered quite comparable results as far as Shannon entropy was concerned. To get more accurate results, it would be necessary to design/employ proper correlation functionals suited for confined systems. 
It was seen that $S_{\mathbf{r}}$ amplified and $S_{\mathbf{p}}$ declined with the rise in $r_{c}$, in both ground and excited states under consideration. Besides, for a particular confinement strength, as $Z$ grew, the state of a system became more localized with a consequent drop and rise in $S_{\mathbf{r}}, S_{\mathbf{p}}$, respectively. For the two families of states arising out of configurations (a) $1 \mathrm{sns}^{3} \mathrm{~S}(\mathrm{n}=2-4)$ and (b) $1 \mathrm{~s} 3 \mathrm{~s}^{3} \mathrm{~S}, 1 \mathrm{~s} 3 \mathrm{p}{ }^{3} \mathrm{P}, 1 \mathrm{~s} 3 \mathrm{~d}{ }^{3} \mathrm{D}$, in the intermediate and lower $r_{c}$ region, the information entropies showed interesting crossovers and finally reached their free atom limit at certain large $r_{c}$. In all cases, the $S_{t}$ bound was maintained. The emergence of these novel characteristics of $S_{\mathbf{r}}, S_{\mathbf{p}}$, and $S_{t}$ makes such information-centric analyses valuable tools for structure and dynamics under a constrained environment. It would be worthwhile to extend the present study to the case of supposedly more realistic penetrable boundaries. Besides, we are also interested in several other information measures like Fisher information, Onicescu energy, complexity, etc., in such systems. Some of these works may be undertaken in the future.

Author Contributions: S.M. contributed in the methodology, program writing, analysis, writing the manuscript and A.K.R. contributed in all these along with editing the manuscript, funding and project administration. All authors have read and agreed to the published version of the manuscript.

Funding: SM is thankful to IISER Kolkata for a Senior Research Fellowship. Financial support from DAE BRNS, Mumbai (Sanction Order 58/14/03/2019-BRNS), is gratefully acknowledged.

Acknowledgments: SM is thankful to IISER Kolkata for a Senior Research Fellowship. Financial support from DAE BRNS, Mumbai (Sanction Order 58/14/03/2019-BRNS), is gratefully acknowledged. Neetik Mukherjee is thanked for the fruitful discussion. We thank the three anonymous referees for their valuable comments and suggestions.

Conflicts of Interest: The authors declare no conflict of interest.

\section{References}

1. Jaskólski, W. Confined many-electron systems. Phys. Rep. 1996, 271, 1-66. [CrossRef]

2. Dolmatov, V.K.; Baltenkov, A.S.; Connerade, J.-P. Structure and photoionization of confined atoms. Radiat. Phys. Chem. 2004, 2004, 417-433. [CrossRef]

3. Sabin, J.; Brändas, E.; Cruz, S. (Eds.) Advances in Quantum Chemistry; Academic Press: New York, NY, USA, 2009; Volume 57, 58.

4. Sen, K.D. (Ed.) Electronic Structure of Quantum Confined Atoms and Molecules; Springer International Publishing: Cham, Switzerland, 2014.

5. Ley-Koo, E. Recent progress in confined atoms and molecules: Superintegrability and symmetry breakings. Rev. Mex. Fís. 2018, 64, 326-363. [CrossRef]

6. Michels, A.; de Boer, J.; Bijl, A. Remarks concerning molecural interaction and their influence on the polarisability. Physica 1937, 1937, 981-994. [CrossRef]

7. Ludeña, E.V. SCF calculations for hydrogen in a spherical box. J. Chem. Phys. 1977, 1977, 468-470. [CrossRef]

8. Marín, J.L.; Cruz, S.A. Enclosed quantum systems: use of the direct variational method. J. Phys. B 1991, 1991, 2899-2907. [CrossRef]

9. Goldman, S.; Joslin, C. Spectroscopic properties of an isotropically compressed hydrogen atom. J. Phys. Chem. 1992, 1992, 6021-6027. [CrossRef]

10. Aquino, N. Accurate energy eigenvalues for enclosed hydrogen atom within spherical impenetrable boxes. Int. J. Quant. Chem. 1995, 1995, 107-115. [CrossRef]

11. Sen, K.D.; Garza, J.; Vargas, R.; Aquino, N. Static dipole polarizability of shell-confined hydrogen atom. Phys. Lett. A 2002, 2002, 299-304. [CrossRef]

12. Laughlin, C.; Burrows, B.L.; Cohen, M. A hydrogen-like atom confined within an impenetrable spherical box. J. Phys. B 2002, 2002, 701-715. [CrossRef]

13. Laughlin, C. On the dipole polarizability of a hydrogen-like atom confined in an impenetrable spherical box. J. Phys. B 2004, 2004, 4085-4099. [CrossRef]

14. Burrows, B.L.; Cohen, M. Exact solutions for perturbed confined hydrogen atoms: Polarizabilities and nuclear shielding factors. Phys. Rev. A 2005, 2005, 032508. [CrossRef]

15. Sen, K.D.; Roy, A.K. Studies on the 3D confined potentials using generalized pseudospectral approach. Phys. Lett. A 2006, 2006, 112-119. [CrossRef] 
16. Burrows, B.L.; Cohen, M. Exact solutions for spherically confined hydrogen-like atoms. Int. J. Quant. Chem. 2006, 2006, 478-484. [CrossRef]

17. Aquino, N.; Campoy, G.; Montgomery, H.E., Jr. Highly accurate solutions for the confined hydrogen atom. Int. J. Quant. Chem. 2007, 2007, 1548-1558. [CrossRef]

18. Baye, D.; Sen, K.D. Confined hydrogen atom by the Lagrange-mesh method: Energies, mean radii, and dynamic polarizabilities. Phys. Rev. E 2008, 2008, 026701. [CrossRef]

19. Ciftci, H.; Hall, R.L.; Saad, N. Study of a confined hydrogen-like atom by the asymptotic iteration method. Int. J. Quant. Chem. 2009, 2009, 931-937. [CrossRef]

20. Montgomery, H.E., Jr.; Sen, K.D. Electron density and its derivatives at the nucleus for spherically confined hydrogen atom. Int. J. Quant. Chem. 2009, 2009, 688-692. [CrossRef]

21. Roy, A.K. Spherical confinement of coulombic systems inside an impenetrable box: $\mathrm{H}$ atom and the hulthén potential Int. J. Quant. Chem. 2015, 2015, 937-947. [CrossRef]

22. Roy, A.K. Critical parameters and spherical confinement of $\mathrm{H}$ atom in screened Coulomb potential. Int. J. Quant. Chem. 2016, 2016, 953-960. [CrossRef]

23. Ten Seldam, C.A.; De Groot, S.R. On the ground state of a model for compressed helium. Physica 1952, 1952, 891-904. [CrossRef]

24. Ludeña, E.V. SCF Hartree-Fock calculations of ground state wavefunctions of compressed atoms. J. Chem. Phys. 1978, 1978, 1770-1775. [CrossRef]

25. Garza, J.; Hernández-Pérez, J.M.; Ramírez, J.-Z.; Vargas, R. Basis set effects on the Hartree-Fock description of confined many-electron atoms. J. Phys. B 2012, 2012, 015002. [CrossRef]

26. Ludeña, E.V.; Gregori, M. Configuration interaction calculations for two-electron atoms in a spherical box. J. Chem. Phys. 1979, 1979, 2235-2240. [CrossRef]

27. Rivelino, R.; Vianna, J.D.M. A configuration interaction model to investigate many-electron systems in cavities. J. Phys. B 2001, 34, L645-L650. [CrossRef]

28. Joslin, C.; Goldman, S. Quantum Monte Carlo studies of two-electron atoms constrained in spherical boxes. J. Phys. B 1992, 1992, 1965-1975. [CrossRef]

29. Banerjee, A.; Kamal, C.; Chowdhury, A. Calculation of ground-and excited-state energies of confined helium atom. Phys. Lett. A 2006, 2006, 121-125. [CrossRef]

30. Flores-Riveros, A.; Aquino, N.; Montgomery, H.E., Jr. Spherically compressed helium atom described by perturbative and variational methods. Phys. Lett. A 2010, 2010, 1246-1252. [CrossRef]

31. Le Sech, C.; Banerjee, A. A variational approach to the Dirichlet boundary condition: Application to confined H-, He and Li. J. Phys. B 2011, 2011, 105003. [CrossRef]

32. Si, T.-Y.; Bao, C.-G.; Li, B.-W. Energy spectra of the confined atoms obtained by using B-splines. Commun. Theor. Phys. 2001, 2001, 195-200.

33. Montgomery, H.E., Jr.; Aquino, N.; Flores-Riveros, A. The ground state energy of a helium atom under strong confinement. Phys. Lett. A 2010, 2010, 2044-2047. [CrossRef]

34. Aquino, N.; Flores-Riveros, A.; Rivas-Silva, J.F. The compressed helium atom variationally treated via a correlated Hylleraas wave function. Phys. Lett. A 2003, 2003, 326-336. [CrossRef]

35. Flores-Riveros, A.; Rodríguez-Contreras, A. Compression effects in helium-like atoms $(Z=1, \ldots, 5)$ constrained by hard spherical walls. Phys. Lett. A 2008, 2008, 6175-6182. [CrossRef]

36. Laughlin, C.; Chu, S.I. A highly accurate study of a helium atom under pressure. J. Phys. A 2009, $2009,265004$. [CrossRef]

37. Wilson, C.L.; Montgomery, H.E., Jr.; Sen, K.D.; Thompson, D.C. Electron correlation energy in confined two-electron systems. Phys. Lett. A 2010, 2010, 4415-4419. [CrossRef]

38. Montgomery, H.E. Jr.; Pupyshev, V.I. Confined helium: excited singlet and triplet states Phys. Lett. A 2013, 2013, 2880-2883. [CrossRef]

39. Bhattacharyya, S.; Saha, J.K.; Mukherjee, P.K.; Mukherjee, T.K. Precise estimation of the energy levels of two-electron atoms under spherical confinement. Phys. Scr. 2013, 2013, 065305. [CrossRef]

40. Montgomery, H.E., Jr.; Pupyshev, V.I. Confined two-electron systems: excited singlet and triplet S states. Theor. Chem. Acc. 2015, 2015, 1598. [CrossRef]

41. Saha, J.; Bhattacharyya, S.; Mukherjee, T.K. Ritz variational calculation for the singly excited states of compressed two-electron atoms. Int. J. Quantum Chem. 2016, 2016, 1802-1813. [CrossRef] 
42. Yakar, Y.; Çakir, B.; Özmen, A. Computation of ionization and various excited state energies ofhelium and helium-like quantum dots. Int. J. Quant. Chem. 2011, 2011, 4139-4149. [CrossRef]

43. Doma, S.B.; El-Gammal, F.N. Application of variational Monte Carlo method to the confined helium atom J. Theor. Appl. Phys. 2012, 2012, 28. [CrossRef]

44. Sarsa, A.; Le Sech, C. Variational Monte Carlo method with dirichlet boundary conditions: Application to the study of confined systems by impenetrable surfaces with different symmetries. J. Chem. Theory Comput. 2011, 2011, 2786-2794. [CrossRef] [PubMed]

45. Young, T.D.; Vargas, R.; Garza, J. A Hartree-Fock study of the confined helium atom: Local and global basis set approaches. Phys. Lett. A 2016, 2016, 712-717. [CrossRef]

46. Pupyshev, V.I.; Montgomery, H.E., Jr. One-and multiconfigurational study of excited states of He atom in a small impenetrable cavity. Theor. Chem. Acc. 2017, 2017, 138. [CrossRef]

47. Parr, R.G.; Yang, W. Density Functional Theory of Atoms and Molecules; Oxford University Press: New York, NY, USA, 1989.

48. Fiolhais, C.; Nogueira, F.; Marques, M. A Primer in Density Functional Theory; Springer: Berlin/Heidelberg, Germany, 2003.

49. Engel, E.; Dreizler, R.M. Density Functional Theory: An Advance Course (Theoretical and Mathematical Physics); Springer: New York, NY, USA, 2011.

50. Becke, A.D. Density-functional exchange-energy approximation with correct asymptotic behavior. Phys. Rev. A 1988, 1988, 3098-3100. [CrossRef]

51. Garza, J.; Vargas, R.; Vela, A. Numerical self-consistent-field method to solve the Kohn-Sham equations in confined many-electron atoms. Phys. Rev. E 1998, 1998, 3949-3954. [CrossRef]

52. Duarte-Alcaráz, F.-A.; Martínez-Sánchez, M.-A.; Rivera-Almazo, M.; Vargas, R.; Rosas-Burgos, R.-A.; Garza, J. Testing one-parameter hybrid exchange functionals in confined atomic systems. J. Phys. B 2019, 2019, 135002. [CrossRef]

53. Aquino, N.; Flores-Riveros, A.; Rivas-Silva, J.F.; Sen, K.D. Confined helium atom low-lying $S$ states analyzed through correlated Hylleraas wave functions and the Kohn-Sham model. J. Chem. Phys. 2006, 2006, 054311. [CrossRef]

54. Perdew, J.P.; Wang, Y. Accurate and simple analytic representation of the electron-gas correlation energy. Phys. Rev. B 1992, 1992, 13244-13249. [CrossRef]

55. Waugh, S.; Chowdhury, A.; Banerjee, A. On the variation of polarizability and hyperpolarizability of a confined atom with the strength of confinement: A case study of a helium atom. J. Phys. B 2010, 2010, 225002. [CrossRef]

56. Vyboishchikov, S.F. Modeling Exact Exchange Potential in Spherically Confined Atoms. J. Comput. Chem. 2015, 2015, 2037-2043. [CrossRef] [PubMed]

57. Lozano-Espinosa, M.; Garza, J.; Galván, M. Confinement effects on the spin potential of first row transition metal cations. Philos. Mag. 2017, 2017, 284-297. [CrossRef]

58. Vyboishchikov, S.F. Correlation energy, correlated electron density, and exchange-correlation potential in some spherically confined atoms. J. Comput. Chem. 2016, 2016, 2677-2686. [CrossRef] [PubMed]

59. Vyboishchikov, S.F. A Simple Local Correlation Energy Functional for Spherically Confined Atoms from ab Initio Correlation Energy Density. ChemPhysChem 2017, 2017, 3478-3484. [CrossRef]

60. van Faassen, M. Atoms in boxes: From confined atoms to electron-atom scattering. J. Chem. Phys. 2009, 2009, 104108.

61. Shannon, C.E. Prediction and Entropy of Printed English. Bell Sys. Tech. J. 1951, 1951, 50-64. [CrossRef]

62. Bialynicki-Birula, I.; Mycielski, J. Uncertainty relations for information entropy in wave mechanics. Commun. Math. Phys. 1975, 1975, 129-132. [CrossRef]

63. Ou, J.-H.; Ho, Y.K. Benchmark calculations of Rényi, Tsallis entropies, and Onicescu information energy for ground state helium using correlated Hylleraas wave functions Int. J. Quantum Chem. 2019, 119, e25928. [CrossRef]

64. Sen, K.D. Characteristic features of Shannon information entropy of confined atoms. J. Chem. Phys. 2005, 2005, 074110. [CrossRef]

65. Jiao, L.G.; Zan, L.R.; Zhang, Y.Z.; Ho, Y.K. Benchmark values of Shannon entropy for spherically confined hydrogen atom. Int. J. Quant. Chem. 2017, 117, e25375. [CrossRef] 
66. Mukherjee, N.; Roy, A.K. Information-entropic measures in free and confined hydrogen atom. Int. J. Quant. Chem. 2018, 118, e25596. [CrossRef]

67. Mukherjee, N.; Roy, A.K. Information-entropic measures for non-zero $l$ states of confined hydrogen-like ions Eur. Phys. J. D 2018, 2018, 118.

68. Aquino, N.; Flores-Riveros, A.; Rivas-Silva, J.F. Shannon and Fisher entropies for a hydrogen atom under soft spherical confinement. Phys. Lett. A 2013, 2013, 2062-2068. [CrossRef]

69. Cruz, C.Díaz-García, S.A.; Olivares-Pilón, H.; Cabrera-Trujillo, R. Many-electron atom confinement by a penetrable planar boundary. Radiat. Effects Defects Solids 2016, 2016, 123-134. [CrossRef]

70. Ou, J.-H.; Ho, Y.K. Shannon information entropy in position space for the ground and singly excited states of helium with finite confinements. Atoms 2017, 2017, 15. [CrossRef]

71. Ou, J.-H.; Ho, Y.K. Shannon information entropy in position space for doubly excited states of helium with finite confinements. Chem. Phys. Lett. 2017, 2017, 116-120. [CrossRef]

72. Rodriguez-Bautista, M.; Vargas, R.; Aquino, N.; Garza, J. Electron-density delocalization in many-electron atoms confined by penetrable walls: A Hartree-Fock study. Int. J. Quant. Chem. 2018, 118, e25571. [CrossRef]

73. Martínez-Sánchez, M.-A.; Vargas, R.; Garza, J. Shannon Entropy for the Hydrogen Atom Confined by Four Different Potentials. Quantum Rep. 2019, 2019, 208-218. [CrossRef]

74. Restrepo Cuartas, J.P.; Sanz-Vicario, J.L. nformation and entanglement measures applied to the analysis of complexity in doubly excited states of helium. Phys. Rev. A 2015, 2015, 052301. [CrossRef]

75. Brual, G.; Rothstein, S.M. Rare gas interactions using an improved statistical method. J. Chem. Phys. 1978, 1978, 1177-1183. [CrossRef]

76. Lee, C.; Wang, Y.; Parr, R.G. Development of the Colle-Salvetti correlation-energy formula into a functional of the electron density. Phys. Rev. B 1988, 1988, 785-789. [CrossRef] [PubMed]

77. Roy, A.K.; Singh, R.; Deb, B.M. Density-functional calculations for doubly excited states of $\mathrm{He} \mathrm{Li}^{+}, \mathrm{Be}^{2+}$ and $\mathrm{B}^{3+}\left(1,3 S^{e},{ }^{3} P^{o}, 1,3 D^{e}, 1,3 P^{o},{ }^{1} G^{e},\right)$. J. Phys. B 1997, 1997, 4763-4782. [CrossRef]

78. Roy, A.K.; Singh, R.; Deb, B.M. Density functional calculations on triply excited states of lithium isoelectronic sequence. Int. J. Quant. Chem. 1997, 1997, 317-332. [CrossRef]

79. Roy, A.K.; Deb, B.M. Atomic inner-shell transitions: A density functional approach. Phys. Lett. A 1997, 1997, 465-471. [CrossRef]

80. Roy, A.K.; Chu, S.I. Density-functional calculations on singly and doubly excited Rydberg states of many-electron atoms. Phys. Rev. A 2002, 2002, 052508. [CrossRef]

81. Roy, A.K. Studies on the hollow states of atomic lithium using a density functional approach. J. Phys. B 2004, 2004, 4369-4386. [CrossRef]

82. Roy, A.K. Density functional studies on the hollow resonances in the $\mathrm{Li}$-isoelectronic sequence $(Z=4-10)$. J. Phys. B. 2005, 2005, 1591-1605. [CrossRef]

83. Roy, A.K.; Jalbout, A.F. Ground and excited states of Li-, Be- through a density-based approach. Chem. Phys. Lett. 2007, 2007, 355-360. [CrossRef]

84. Sahni, V.; Harbola, M. Quantum-Mechanical interpretation of the local many-body potential of density-functional theory. Int. J. Quant. Chem. Symp. 1990, 1990, 569-584. [CrossRef]

85. Sahni, V.; Li, Y.; Harbola, M. Atomic structure in the Pauli-correlated approximation. Phys. Rev. A 1992, 1992, 1434-1448. [CrossRef]

86. Roy, A.K. Calculation of the spiked harmonic oscillators through a generalized pseudospectral method. Phys. Lett. A 2004, 2004, 231-238. [CrossRef]

87. Roy, A.K. Calculation of the bound states of power-law and logarithmic potentials through a generalized pseudospectral method. J. Phys. G, 2004, 2004, 269-278. [CrossRef]

88. Roy, A.K. The generalized pseudospectral approach to the bound states of Húlthen and Yukawa potential. Pramana J. Phys. 2005, 2005, 1-15. [CrossRef]

89. Roy, A.K. Studies on some singular potentials in quantum mechanics. Int. J. Quant. Chem. 2005, 2005, 861-870. [CrossRef]

90. Guevara, N.L.; Sagar, R.P.; Esquivel, R.O. Information uncertainty-type inequalities in atomic systems. J. Chem. Phys. 2003, 2003, 7030-7036. [CrossRef]

91. Gadre, S.R.; Sears, S.B.; Chakravorty, S.J.; Bendale, R.D. Some novel characteristics of atomic information entropies. Phys. Rev. A 1985, 1985, 2602-2606. [CrossRef] 
92. Amovilli, C.; Floris, F.M. Shannon entropy in atoms: a test for the assessment of density functionals in Kohn-Sham theory. Computation 2018, 2018, 36. [CrossRef]

93. Lin, C.-H.; Ho, Y.K. Shannon information entropy in position space for two-electron atomic systems. Chem. Phys. Lett. 2015, 2015, 261-264. [CrossRef]

94. Lin, C.-H.; Ho, Y.K. Shannon, Rényi, Tsallis Entropies and Onicescu Information Energy for Low-Lying Singly Excited States of Helium. Atoms 2019, 2019, 70.

(C) 2020 by the authors. Licensee MDPI, Basel, Switzerland. This article is an open access article distributed under the terms and conditions of the Creative Commons Attribution (CC BY) license (http:/ / creativecommons.org/licenses/by/4.0/). 